\title{
Mudança significativa do limiar auditivo em trabalhadores expostos a diferentes níveis de ruído
}

\author{
Significant auditory threshold shift among workers exposed to \\ different noise levels
}

\author{
Flavia Cardoso Oliva ${ }^{1}$, Thais Catalani Morata ${ }^{2}$, Adriana Bender Moreira de Lacerda ${ }^{2}$, Luciara Steinmetz ${ }^{1}$, \\ Luciana Bramatti ${ }^{1}$, Ane Gleise Vivan¹, Claudia Giglio de Oliveira Gonçalves ${ }^{2}$, Jair Mendes Marques²
}

\begin{abstract}
RESUMO
Objetivo: Avaliar a audição e a ocorrência de mudança significativa do limiar auditivo em trabalhadores de frigoríficos expostos a níveis de ruído abaixo das Normas e Regulamentações nacionais e internacionais e compará-los com trabalhadores expostos a níveis de ruído considerados excessivos. Métodos: Utilizou-se um banco de dados contendo informações longitudinais de 266 trabalhadores. Foram selecionados trabalhadores com um mínimo de três exames audiométricos e os que continham dados de exposição ao ruído. Foram mantidos 63 exames, classificados de acordo com sua exposição ao ruído em três níveis: 79 a 84,9 dB(A), 85 a 89,9 dB(A) e 90 a 98,8 dB(A). Foi avaliada a ocorrência de perdas auditivas e de mudança significativa de limiar auditivo dos participantes de cada subgrupo. Resultados: Verificou-se diferenças em todas as frequências nos testes de comparação entre a média dos limiares auditivos para cada frequência em função do nível de exposição ao ruído. A correlação entre a ocorrência de Perda Auditiva Induzida por Níveis de Pressão Sonora Elevados (PAINPSE) e os anos de exposição ao ruído dentro da empresa atual foi significativa (R=0,373; $\mathrm{p}=0,079$ ). Foram encontradas mudanças permanentes de limiar auditivo nos três níveis de exposição ao ruído. Conclusão: Os achados do presente estudo sugerem a existência de uma associação entre mudança significativa do limiar auditivo dos trabalhadores e os anos de exposição ao ruído considerado de baixo risco.
\end{abstract}

Descritores: Limites permissíveis; Legislação como assunto; Perda auditiva provocada por ruído; Exposição ocupacional; Ruído ocupacional

\section{INTRODUÇÃO}

O ruído é o principal agente físico presente em diferentes categorias profissionais. A exposição a níveis de pressão sonora elevados em função de sua duração, frequência, intensidade e suscetibilidade individual, além de acarretar múltiplas consequências ao organismo humano, constitui um dos principais riscos à audição, podendo causar a perda auditiva por níveis de pressão sonora elevados ${ }^{(1)}$.

Com o intuito de regulamentar a exposição dos trabalhadores ao ruído intenso, evitando a surdez ocupacional, a legislação, em todo o mundo, adota critérios estabelecendo formas de intervenções baseadas em ações preventivas das

Trabalho realizado no Curso de Fonoaudiologia, Universidade Tuiuti do Paraná - UTP - Curitiba (PR), Brasil.

(1) Programa de Pós-graduação (Mestrado) em Distúrbios da Comunicação Humana, Universidade Tuiuti do Paraná - UTP - Curitiba (PR), Brasil.

(2) Programa de Pós-graduação em Distúrbios da Comunicação Humana, Universidade Tuiuti do Paraná - UTP - Curitiba (PR), Brasil.

Endereço para Correspondência: Claudia Giglio de Oliveira Gonçalves. R. Sydney Antonio Rangel Santos, 238, Santo Inácio, Curitiba (PR), Brasil, CEP: 82010-330. E-mail: claudia.goncalves@utp.br

Recebido em: 20/5/2009; Aceito em: 19/10/2010 alterações auditivas de origem ocupacional. Assim sendo, no Brasil e em muitos outros países, para a regulamentação das condições dos ambientes de trabalho, utiliza-se do modelo do Limite de Tolerância (LT), que corresponde à concentração ou à intensidade mínima ou máxima de agentes de risco a que o trabalhador pode se expor. $\mathrm{O}$ dano causado pela exposição aos agentes de risco é determinado pelo tempo de exposição em horas diárias e em anos, durante a vida laboral do trabalhador. Para alguns autores, o limite de tolerância não deve ser compreendido como a linha que identifica a existência de risco, mas como definidor da magnitude de risco aceitável ${ }^{(2)}$.

O risco de desenvolver perda auditiva em razão de exposição a ruído no ambiente de trabalho aumenta conforme o tempo de exposição em anos. Daí a necessidade do controle do ruído e do monitoramento auditivo anual. Entende-se como monitoramento auditivo a análise dos exames auditivos sequenciais comparados com o exame de referência, possibilitando a tomada de decisões em relação à audição do trabalhador ${ }^{(1)}$.

O LT para o agente ruído foi reconhecido pela legislação brasileira e, tecnicamente, aceito como limite de exposição ocupacional em 1978. O Brasil baseou-se na recomendação da Conferência Americana dos Higienistas Industriais 
governamentais (American Conference of Governmental Industrial Hygienists - ACGIH) em $1977^{(3)}$, adotando o nível de $85 \mathrm{~dB}(\mathrm{~A})$ e o incremento de duplicação de dose (q) igual a $5 \mathrm{~dB}$ para o cálculo da exposição diária permissível. Entende-se como incremento de duplicação de dose o valor em dB que dobra o risco para a saúde, então, o tempo de exposição permitida reduz-se pela metade. Num q=5, a cada aumento de $5 \mathrm{~dB}$ na intensidade sonora, diminui pela metade o tempo permitido da exposição do trabalhador, então, se a $85 \mathrm{~dB}$ pode-se expor o trabalhador a oito horas, quando em $90 \mathrm{~dB}$ a exposição permitida é de quatro horas. Nessa época, a ACGIH diferenciava-se da Norma Regulamentadora 15 (Brasil, 1978) ${ }^{(4)}$ apenas no limite máximo de exposição que era de $90 \mathrm{~dB}(\mathrm{~A})$. Posteriormente, a ACGIH alterou o limite permissível de exposição de $85 \mathrm{~dB}(\mathrm{~A})$ para oito horas com incremento de duplicação de dose de $3 \mathrm{~dB}(\mathrm{~A})$. Atualmente, a agência governamental responsável pela saúde e proteção ocupacional dos trabalhadores americanos a Ocupational Safety and Health Administration (OSHA) ${ }^{(5)}$, ainda considera o limite de exposição máximo ao ruído de $90 \mathrm{~dB}(\mathrm{~A})$, com incremento de duplicação de dose (também chamado de fator de conversão, ou de dobra de dose) de $5 \mathrm{~dB}(\mathrm{~A})$.

Com exceção do Brasil, Chile, Israel, Estados Unidos e Colômbia, que ainda utilizam incremento de duplicação de dose de $5 \mathrm{~dB}$, diversos países adotaram incremento de duplicação de dose de $3 \mathrm{~dB}^{(6)}$. É importante ressaltar que a utilização de um fator de conversão menor implica a necessidade de inclusão de um maior número de empregados nos programas para prevenção de perdas auditivas.

A União Européia publicou, em 2003, uma nova diretriz, 2003/10/CE ${ }^{(7)}$, que estabelece prescrições mínimas de proteção aos trabalhadores nos países membros contra os riscos da exposição ao ruído, especialmente contra riscos para a audição. A nova diretriz estabeleceu um limite de exposição permissível de $87 \mathrm{~dB}(\mathrm{~A})$ para oito horas trabalhadas, com um incremento de duplicação de dose de $3 \mathrm{~dB}$, levando-se em conta a atenuação proporcionada pelos protetores auditivos para a determinação da exposição sonora efetiva do trabalhador.

No Brasil, a Fundacentro ${ }^{(8)}$ segue a tendência mundial, pois recomenda um limite de exposição ao ruído de $85 \mathrm{~dB}(\mathrm{~A})$ para uma exposição de oito horas diárias, com um incremento de duplicação de dose (q) de $3 \mathrm{~dB}(\mathrm{~A})$, sendo que, nesse critério, o nível de ação inicia em $82 \mathrm{~dB}(\mathrm{~A})(\mathrm{NHO}-1)^{(8)}$.

Diante das Normas e recomendações nacionais (NHO1) e internacionais ${ }^{(9)}$ existentes, o Limite de Tolerância para exposição ao ruído no Brasil necessita de uma reavaliação, na tentativa de adequação da legislação atual, visando a proteção da audição dos trabalhadores.

Dentre as diversas atividades que expõem os trabalhadores a níveis elevados de pressão sonora encontra-se a atividade nos frigoríficos, que, entre outros riscos para a saúde, apresenta ambientes com ruído contínuo acima de $80 \mathrm{~dB}(\mathrm{~A})$, podendo causar perdas auditivas ocupacionais ${ }^{(10)}$.

O objetivo desse estudo é avaliar a audição e a ocorrência de mudança significativa do limiar auditivo em trabalhadores que estão expostos a níveis de ruído ocupacional considerados de baixo risco para perda auditiva e comprá-los ao grupo de trabalhadores expostos a níveis de ruído considerados excessivos.

\section{MÉTODOS}

\section{Caracterização da amostra}

O presente trabalho foi aprovado pelo Comitê de Ética da Universidade Tuiuti do Paraná (UTP), sob o protocolo de número 0084/2007.

Para este estudo - uma pesquisa observacional e de corte retrospectivo - utilizou-se o banco de dados ocupacionais do núcleo de trabalho "Saúde Auditiva: enfoque preventivo", do Programa de Mestrado e Doutorado em Distúrbios da Comunicação da Universidade Tuiuti do Paraná (UTP).

O banco de dados é constituído por 266 trabalhadores de indústrias frigoríficas, cuja atividade principal é o abate de frangos, totalizando 682 exames. Este banco possui informações sobre a história ocupacional (função, tempo na empresa, nível de ruído, tipo de protetor auricular, tempo de exposição ao ruído) e auditiva (queixa de perda auditiva, queixa de zumbido, tempo de zumbido, hobbies ruidosos), bem como os resultados das avaliações auditivas dos funcionários.

Estabeleceu-se, como critério de inclusão, que cada participante deveria ter, no mínimo, três exames audiométricos e informações sobre o nível de ruído a que estava exposto; dessa forma 63 indivíduos (419 audiometrias) foram investigados e divididos em 3 grupos, de acordo com o nível de exposição ao ruído:

- Nível 1: composto por 28 indivíduos (185 audiometrias) expostos ao nível de ruído na faixa entre 79 e $84,9 \mathrm{~dB}(\mathrm{~A})$ (nível médio de 82,1 dB(A) e desvio padrão de 1,12), sendo que o primeiro exame da série foi o admissional em 14 dos casos;

- Nível 2: composto por 27 indivíduos (167 audiometrias) expostos ao nível de ruído na faixa entre 85 e $90 \mathrm{~dB}(\mathrm{~A})$ (nível médio de 87,4 $\mathrm{dB}(\mathrm{A})$ e desvio padrão de 1,76), sendo que para 16 destes o primeiro exame da série foi o admissional;

- Nível 3: composto por oito indivíduos (67 audiometrias) expostos ao nível de ruído na faixa de 90 e $98,8 \mathrm{~dB}(\mathrm{~A})$ (nível médio de 94,2 dB(A) e desvio padrão de 2,76), sendo que, para um destes, o primeiro exame da série foi o admissional.

É importante ressaltar que alguns participantes desse estudo já apresentavam perda auditiva induzida por ruído no primeiro exame, em diferentes estágios de progressão.

\section{Procedimentos}

O nível de ruído foi avaliado pelo Setor de Engenharia de Segurança da empresa, utilizando-se Audio Dosímetro 897 Simpson, com áudio calibrador operando em circuito de compensação "A" e circuito de resposta lenta (slow). Todas as medições foram realizadas próximas à orelha do trabalhador, conforme Portaria $\mathrm{n}^{0}$ 3.214/78 do Ministério do Trabalho ${ }^{(3)}$. Todos os funcionários incluídos no estudo tinham acesso a protetores auditivos do tipo concha. $\mathrm{O}$ uso dos protetores auditivos era exigido e fiscalizado em toda a área fabril, independente do nível de exposição ao qual o funcionário estava exposto. 
Para as avaliações auditivas, foram respeitados os critérios sugeridos pela NR 7 (Portaria n ${ }^{\circ} 19$ da NR-7 - MTb, Brasil, 1998) $)^{(11)}$ :

a) inspeção do meato acústico, para investigar a presença de cerume;

b) audiometria tonal liminar em cabine acústica Vibrasom $₫$ sob repouso acústico de 14 horas, audiômetro da marca Interacoustics ${ }^{\circledR}$, modelo AD 229 e, com fone TDH-39 calibrado de acordo com as normas internacionais. As frequências avaliadas por via aérea foram de $250 \mathrm{~Hz}$ a 8 $\mathrm{kHz}$ e, quando o limiar encontrado fosse maior que $25 \mathrm{~dB}$, realizava-se por via óssea de 500 a $4 \mathrm{kHz}$. As audiometrias foram classificadas conforme critério da Portaria $n^{\circ} 19$ do Ministério do Trabalho (Brasil, 1998) ${ }^{(11)} \mathrm{em:}$

- audiograma dentro dos limites aceitáveis (compatível com a normalidade): quando os limiares tonais em todas as frequências apresentarem valores inferiores ou iguais a 25 dBNA. - audiograma sugestivo de Perda Auditiva Induzida por Níveis de Pressão Sonora Elevados (PAINPSE): acometimento neurossensorial na forma de entalhe nas frequências altas (3 e/ou 4 e/ou $6 \mathrm{kHz}$ ), maiores ou iguais a $30 \mathrm{~dB}$;

- audiograma não sugestivo de PAINPSE, sugestivo de outras causas auditivas não associadas ao ruído: audiograma não característico de entalhe nas frequências altas. Incluem-se aqui os audiogramas com prováveis ocorrências concomitantes de PAINPSE e com outras alterações auditivas.

Para o monitoramento auditivo, a mudança significativa de limiar foi analisada considerando-se os critérios estabelecidos na Norma Regulamentar n. 7 (NR-7) Anexo 1 do Ministério do Trabalho $^{(11)}$ e o critério de $15 \mathrm{~dB}$ TWICE (ou seja, confirmado em reteste) da National Institute for Occupational Safety and Health - NIOSH $(1998)^{(8)}$. O critério de $15 \mathrm{~dB}$ TWICE do NIOSH (1998) ${ }^{(9)}$ considera que a mudança de $15 \mathrm{~dB}$ ou mais em qualquer frequência testada de $500 \mathrm{~Hz}$ a $6 \mathrm{kHz}$ deve ser persistente na mesma frequência e na mesma orelha no próximo exame realizado.

\section{Estatística}

Utilizou-se o teste ANOVA para a verificação da diferença estatística da média da exposição na empresa e para a média dos limiares auditivos para cada grupo de trabalhadores expostos a cada um dos três níveis de ruído, com nível de significância de 0,05 (5\%).

O teste T-Student para dados pareados permitiu a comparação dos limiares médios por frequência dos primeiros e últimos exames da série $(n=63)$ nos três níveis de exposição estudados.

Utilizou-se a correlação de Spearman e o correspondente teste de significância dos níveis de ruído (1,2 e 3: n=63), para correlacionar a idade com o tempo de trabalho na empresa atual, com nível de significância de 0,05.

\section{RESULTADOS}

Na Tabela 1 estão descritas as características da população estudada segundo a idade e o gênero em função do nível de exposição ao ruído e a média dos anos de exposição da população estudada $(n=63)$.

Comparando-se os três grupos em relação à média de idade e à média de exposição ao ruído na empresa, por meio do teste ANOVA, verifica-se que não houve diferença entre os mesmos ao nível de significância de $5 \%$ ( $\mathrm{p}=0,491$ e $\mathrm{p}=0,375$, respectivamente). A única correlação que se aproximou ao nível significativo foi entre a ocorrência de PAINPSE e os anos de exposição ao ruído dentro da empresa atual $(\mathrm{R}=0,373$; $\mathrm{p}=0,079$ ).

Observou-se a queixa de zumbido em apenas dois sujeitos, sendo que um deles exposto à faixa de 79 a 84,9 $\mathrm{dB}(\mathrm{A})$ e o outro no grupo exposto à faixa de 85 a 89,9 dB(A). Em relação à queixa de perda auditiva, foi relatada por quatro sujeitos, sendo que três deles do grupo exposto à faixa de 79 a 84,9 $\mathrm{dB}(\mathrm{A})$ e um exposto à faixa de 85 a 89,9 dB(A).

Em relação ao perfil auditivo da população estudada, encontrou-se $32,1 \%$ dos audiogramas sugestivos de PAINPSE no grupo exposto a ruído do Nível $1,44,5 \%$ no grupo do nível 2 e $37,5 \%$ no grupo do nível 3.

Na Tabela 2 podem-se observar os resultados da comparação do limiar auditivo médio, por orelha, do primeiro e último exame realizado pelos sujeitos estudados. Os resultados descritos abaixo apresentam significância estatística maior no Nível 2, porém o Nível 1 mostra que a orelha esquerda também apresentou diferença entre os exames analisados.

Na Tabela 3, resume-se o resultado dos testes de comparação entre a média dos limiares auditivos de toda a população, para cada frequência, em função do nível de exposição ao

Tabela 1. Características da população estudada segundo a idade e gênero em relação ao nível de exposição ao ruído ( $\mathrm{n}=63$ )

\begin{tabular}{|c|c|c|c|c|c|c|c|c|c|c|c|c|}
\hline \multirow{3}{*}{$\begin{array}{l}\text { Variáveis } \\
\text { Idade (em anos) }\end{array}$} & \multicolumn{4}{|c|}{$\begin{array}{c}\mathrm{NR} 1(\mathrm{n}=28) \\
79 \text { a } 84,9 \mathrm{~dB}(\mathrm{~A})\end{array}$} & \multicolumn{4}{|c|}{$\begin{array}{c}\mathrm{NR} 2(\mathrm{n}=27) \\
85 \text { a } 89,9 \mathrm{~dB}(\mathrm{~A})\end{array}$} & \multicolumn{4}{|c|}{$\begin{array}{c}\text { NR } 3(n=8) \\
90 \text { a } 98,8 d B(A)\end{array}$} \\
\hline & \multicolumn{2}{|c|}{ Média } & \multicolumn{2}{|c|}{ DP } & \multicolumn{2}{|c|}{ Média } & \multicolumn{2}{|c|}{ DP } & \multicolumn{2}{|c|}{ Média } & \multicolumn{2}{|c|}{ DP } \\
\hline & \multicolumn{2}{|c|}{31,4} & \multicolumn{2}{|c|}{8} & \multicolumn{2}{|c|}{29,4} & \multicolumn{2}{|c|}{7} & \multicolumn{2}{|c|}{32,6} & \multicolumn{2}{|c|}{11} \\
\hline & & & & ino & $\mathrm{Ma}$ & lino & & ino & & lino & & \\
\hline \multirow[t]{2}{*}{ Gênero } & $n$ & $\%$ & $\mathrm{n}$ & $\%$ & $n$ & $\%$ & $\mathrm{n}$ & $5 \%$ & $\mathrm{n}$ & $5 \%$ & $n$ & $5 \%$ \\
\hline & 20 & 1,4 & 8 & 8,6 & 21 & 7,8 & 6 & 2,2 & 1 & 2,5 & 7 & 7,5 \\
\hline
\end{tabular}

Tempo médio de exposição ao

ruído na empresa (em anos)

14

9

23

Legenda: $\mathrm{NR}$ = nível de ruído; $\mathrm{DP}$ = desvio-padrão 
Tabela 2. Comparação dos limares auditivos médios, por grupo de exposição, do primeiro e último exame do banco de dados $(n=126)$, por orelha

\begin{tabular}{lcccccc}
\hline $\begin{array}{l}\text { Frequência } \\
(\mathrm{Hz})\end{array}$ & \multicolumn{2}{c}{$\mathrm{NR} 1$} & \multicolumn{2}{c}{$\mathrm{NR} 2$} & \multicolumn{2}{c}{$\mathrm{NR} 3$} \\
\hline 500 & OD & OE & OD & OE & OD & OE \\
1000 & $0,0105^{*}$ & $0,0045^{*}$ & $0,0044^{*}$ & $0,0010^{*}$ & $0,0412^{*}$ & $0,0331^{*}$ \\
2000 & 0,1676 & 0,3712 & 0,6213 & 0,3325 & 0,1334 & 0,5983 \\
3000 & 1,0000 & 0,7513 & 0,1071 & $0,0531^{*}$ & 0,2910 & 0,2849 \\
4000 & 0,9377 & $0,0008^{*}$ & $0,0343^{*}$ & $0,0268^{*}$ & 0,9064 & 0,1211 \\
6000 & 0,3049 & $0,0423^{*}$ & $0.0030^{*}$ & $0,0110^{*}$ & $0,0185^{*}$ & 0,2470 \\
8000 & 0,3550 & $0,0224^{*}$ & $0,0074^{*}$ & $0,0013^{*}$ & 0,6622 & 0,8565 \\
\hline
\end{tabular}

*Valores significativos $(p \leq 0,05)$ - Teste t Student

Legenda: $\mathrm{NR}=$ nível de ruído; $\mathrm{OD}$ = orelha direita; $\mathrm{OE}=$ orelha esquerda

ruído, para observar as mudanças nos limiares auditivos aéreos de um exame para outro. Por meio do teste ANOVA verificase que existe diferença significativa em todas as frequências. Esta tabela mostra também quais são os grupos que diferem entre si, em cada frequência, por orelha.

Tabela 3. Comparação dos limiares médios do total de audiogramas da série ( $n=63 / 419$ audiometrias) em função do nível de exposição ao ruído

\begin{tabular}{lcccc}
\hline $\begin{array}{l}\text { Frequência } \\
(\mathrm{Hz})\end{array}$ & Valor de $\mathrm{p}$ & $\begin{array}{c}\text { Níveis que } \\
\text { diferem }\end{array}$ & Valor de $\mathrm{p}$ & $\begin{array}{c}\text { Níveis que } \\
\text { diferem }\end{array}$ \\
\hline 500 & 0,0063 & 1 e 2, 2 e 3 & 0,0118 & 2 e 3 \\
1000 & 0,0000 & Todos entre si & 0,0000 & Todos entre si \\
2000 & 0,0000 & Todos entre si & 0,0000 & 1 e 3, 2 e 3 \\
3000 & 0,0000 & Todos entre si & 0,0006 & 1 e 3, 2 e 3 \\
4000 & 0,0000 & 1 e 3, 2 e 3 & 0,0000 & 1 e 3, 2 e 3 \\
6000 & 0,0000 & Todos entre si & 0,0000 & 1 e 3, 2 e 3 \\
8000 & 0,0000 & 1 e 2, 2 e 3 & 0,0285 & 1 e 2 \\
\hline
\end{tabular}

* Valores significativos $(\mathrm{p} \leq 0,05)$ - Teste ANOVA

Legenda: $O D$ = orelha direita; $O E$ = orelha esquerda

Realizou-se, em cada nível de ruído, e com cada orelha, o cálculo de ocorrência de mudança significativa de limiar (MSL) e a média tritonal de 3, 4, $6 \mathrm{kHz}$, segundo NIOSH (1998), e a NR7 - Anexo 1, respectivamente, excluindo as perdas não-ocupacionais (de outras configurações que não fossem de alta frequência, ou bilaterais) como demonstra a Tabela 4.

\section{DISCUSSÃO}

Para a análise entre os grupos, controlaram-se as variáveis idade e tempo de exposição ao ruído, que são similares.

As queixas de zumbido e sensação de perda auditiva foram relatadas apenas pelos sujeitos expostos a ruído do nível $1 \mathrm{e}$ do nível 2. Esses achados podem indicar que nos grupos com exposição de 79,9 até 89,9 dB(A) (nível 1 e nível 2) já exista um início de desordem auditiva, mostrando a sua vulnerabilidade em relação ao ruído ${ }^{(12,27)}$.
Tabela 4. Distribuição dos trabalhadores com mudança significativa de limiar (MSL) segundo NIOSH e NR7 - Anexo 1 para perdas ocupacionais $(n=63)$, por orelha

\begin{tabular}{lcccccc}
\hline \multirow{2}{*}{ Níveis de ruído } & \multicolumn{2}{c}{$\mathrm{NR} 1(\mathrm{n}=28)$} & \multicolumn{2}{c}{$\mathrm{NR} 2(\mathrm{n}=27)$} & \multicolumn{2}{c}{$\mathrm{NR} 3(\mathrm{n}=8)$} \\
& 79 a 84,9 dB(A) & 85 a $89,9 \mathrm{~dB}(\mathrm{~A})$ & 90 a $98,8 \mathrm{~dB}(\mathrm{~A})$ \\
& OD & OE & OD & OE & OD & OE \\
\hline \multirow{2}{*}{ NIOSH } & 3 & 2 & 5 & 6 & 1 & 2 \\
NR 7 Anexo 1 & $(11 \%)$ & $(7 \%)$ & $(18 \%)$ & $(22 \%)$ & $(12 \%)$ & $(25 \%)$ \\
& 2 & 2 & 4 & 6 & 1 & 2 \\
\hline
\end{tabular}

Legenda: $\mathrm{NR}=$ nível de ruído; $\mathrm{OD}=$ orelha direita; $\mathrm{OE}$ = orelha esquerda

A comparação dos limiares audiométricos médios do primeiro audiograma da série com os últimos da série revelou que houve agravamento significativo dos limiares, com o passar do tempo, mesmo no grupo com exposição abaixo dos limites de tolerância, na orelha esquerda. Este estudo corrobora, assim, os achados americanos ${ }^{(13)}$ que demonstram a ocorrência de agravamento de limiar entre trabalhadores expostos a ruídos iguais ou menores que os níveis de ação americano, ou seja, $85 \mathrm{~dB}(\mathrm{~A})$, maior do que nos grupos expostos a níveis mais elevados de ruído.Os autores destacaram que a alta taxa de agravamento auditivo em grupos expostos a níveis de ruído iguais ou inferiores a $85 \mathrm{~dB}(\mathrm{~A})$ pode ocorrer em função do uso inconsistente e incorreto dos protetores auditivos nesses ambientes, pois esses trabalhadores receberiam menor atenção por parte da equipe de saúde e segurança do que outros trabalhadores, expostos a níveis mais elevados de ruído.

O grupo que apresentou limiares estatisticamente diferentes foi o exposto aos níveis de ruído entre 85 e $90 \mathrm{~dB}(\mathrm{~A})$. Neste grupo observou-se maior quantidade de agravamento auditivo bilateral entre o primeiro e o último exame. Vários estudos corroboram este achado, pois o nível de ruído destacado está presente em várias empresas e é responsável por alterações auditivas em funcionários de vários segmentos industriais ${ }^{(14,15)}$.

O limiar audiométrico médio do primeiro audiograma da série demonstra que alguns participantes já possuíam alterações auditivas. No banco de dados usado não existe a informação de quantos anos a pessoa esteve exposta ao ruído antes de realizar o primeiro exame. Sabe-se que metade dos primeiros exames da série do subgrupo do Nível 1 (ruído entre 79 e 84,9 dB(A)) eram exames admissionais, enquanto que só 12\% dos primeiros exames do subgrupo Nível 3 (ruído entre 90 e $98,8 \mathrm{~dB}(\mathrm{~A})$ ) eram admissionais. Isso talvez explique por que os limiares médios dos primeiros exames do subgrupo nível 3 foram piores que os outros grupos estudados. Pode explicar também por que foram menores as pioras de limiar nesse grupo, pois se sabe que nos primeiros anos de exposição é que ocorrem as maiores mudanças de limiar ${ }^{(16,17)}$. Apesar dessa limitação, essa comparação ilustra uma piora dos limiares auditivos para os três subgrupos (Níveis 1, 2 e 3) estudados, o que vem sugerir que mesmo o subgrupo exposto a níveis abaixo de $85 \mathrm{~dB}(\mathrm{~A})$ por oito horas está sujeito a piora auditivas.

Também foi encontrado, em um estudo brasileiro, piora dos limiares auditivos em empregados de um hospital geral divididos em grupos expostos e não-expostos a elevados níveis de ruído ${ }^{(15)}$. Dos 114 empregados expostos a níveis de ruído superiores a $81 \mathrm{~dB}(\mathrm{~A}), 60(52,7 \%)$ apresentaram perda auditiva sugestiva 
de PAINPSE e 54 (47,3\%), audição normal. Em relação aos 73 empregados expostos a níveis de ruído inferiores a $80 \mathrm{~dB}(\mathrm{~A})$, constatou-se que 35 (48\%) tinham perda auditiva. O estudo demonstrou que as perdas auditiva ocorreram em empregados expostos a níveis de ruído inferiores a $80 \mathrm{~dB}(\mathrm{~A})$, do gênero masculino, apresentando idades entre 20 e 40 anos e do gênero feminino, apresentando idade superior a 41 anos. Os autores sugeriram que os achados nem sempre correspondem à expectativa de que maiores perdas estariam associadas à exposição ao ruído.

Os critérios utilizados para a análise da mudança significativa de limiar foram o proposto pelo NIOSH $(1998)^{(9)} \mathrm{e}$ pela NR7 - Anexo 1, do Ministério do Trabalho e Emprego ${ }^{(11)}$. Neste estudo, a ocorrência de mudança significativa de perdas auditivas por nível de ruído, foi mais elevada do que aquela descrita pelo NIOSH $(1998)^{(9)}$, ou seja, no estudo realizado por NIOSH, estimou-se a porcentagem de perda auditiva após 40 anos de exposição aos níveis de ruído diários que seguem, respectivamente: 80,85 ou $90 \mathrm{~dB}(\mathrm{~A})$. Segundo o $\mathrm{NIOSH}^{(9)}$ as porcentagens de MSL observadas na exposição de $80 \mathrm{~dB}(\mathrm{~A})$ seriam de 0 a $5 \%$, em $85 \mathrm{~dB}(\mathrm{~A})$ seriam de 15 a $16 \%$ e finalmente na exposição acima de $90 \mathrm{~dB}(\mathrm{~A})$ seriam de 21 a $29 \%$.É importante destacar que o estudo de NIOSH leva em conta toda uma vida de trabalho, ou seja, 40 anos, já em nosso estudo os sujeitos apresentam média máxima de tempo de exposição de 14 anos trabalhados e mostraram uma MSL consideravelmente maior que as encontradas por NIOSH.

Com a informação sobre a combinação da exposição diária e do tempo trabalhado é possível predizer a típica perda auditiva induzida por ruído de qualquer exposição, porém a suscetibilidade não é padrão entre os indivíduos e existe uma grande variação da perda média esperada ${ }^{(18,19)}$. Vários autores mencionam e usam normas internacionais que permitem calcular, através de uma fórmula, a expectativa de perda auditiva em uma população adulta exposta diariamente ao ruído ${ }^{(20-22)}$.A perda auditiva induzida por ruído mostra um entalhe típico na frequência audiométrica de $4 \mathrm{kHz}^{(23)}$ e as fórmulas internacionais refletem essa característica. Em um indivíduo, do gênero masculino, que se expôs ao ruído durante 45 anos, a mudança do limiar médio esperado em $4 \mathrm{kHz}$ deve variar de acordo com esse nível de ruído. Por exemplo, para o nível de ruído de 80
$\mathrm{dB}(\mathrm{A})$ a mudança de limiar esperada é de 2 dBNA, para 85 $\mathrm{dB}(\mathrm{A})$ é de $7 \mathrm{dBNA}$, para $90 \mathrm{~dB}(\mathrm{~A})$ é 15 de dBNA e para 95 $\mathrm{dB}(\mathrm{A})$ é de 27 dBNA. Os achados de nosso estudo mostram MSL maior para os mesmos níveis de exposição supracitados.

Atualmente, supõe-se que a mudança significativa de limiar é mínima quando há exposição a $80 \mathrm{~dB}(\mathrm{~A})$, existe um risco pequeno em $85 \mathrm{~dB}(\mathrm{~A})$ e a partir de $90 \mathrm{~dB}(\mathrm{~A})$ esse risco torna-se importante. Entretanto, os achados de nosso estudo e de outros estudos $^{(15,24)}$ não corroboraram esta suposição.

Devemos levar em consideração que o incremento de duplicação de dose de $5 \mathrm{~dB}$ utilizado atualmente no Brasil é menos conservador que o de $3 \mathrm{~dB}$, o que aumenta a probabilidade de risco de danos auditivos. Vários institutos e pesquisadores recomendam o uso do fator mais conservador de $3 \mathrm{~dB}^{(6,25-27)}$. Entretanto, não podemos esquecer o número reduzido de participantes no presente estudo, e recomendamos que ele seja replicado com bancos de dados maiores.

O banco de dados utilizado foi obtido de uma empresa que orienta e tenta garantir o uso de protetores auditivos entre seus funcionários, porém ainda foi constatada a ocorrência de MSL. Essa constatação sugere que empresas em que tais medidas não sejam empregadas, a ocorrência de MSL poderá ser ainda maior.

Nossos achados sugerem que trabalhadores expostos a níveis de ruído abaixo das Normas e Regulamentações nacionais e internacionais devem receber orientação e treinamento tanto quanto os expostos a níveis superiores de ruído, atuando, desta maneira, preventivamente em possíveis alterações que poderiam ser evitadas.

\section{CONCLUSÃO}

Os resultados do presente estudo demonstram uma correlação significativa entre a ocorrência de PANPSE e os anos de exposição ao ruído dentro da empresa atual. Houve piora do limiar auditivo nos três grupos de trabalhadores expostos ao ruído estudado, mesmo nos trabalhadores cuja exposição encontrava-se abaixo dos limites de exposição permissíveis no Brasil. Destacamos que os resultados obtidos fornecem informações importantes para a continuidade dos estudos nessa área.

\begin{abstract}
Purpose: To assess the hearing status and signs of significant auditory threshold shifts in meat-processing facility workers who are exposed to noise levels below nationally and internationally recommended exposure limits, and to compare these results with data from workers exposed to excessive noise levels. Methods: Longitudinal audiometric data from 266 workers were evaluated, and only workers with a minimum of three audiometric test results and noise exposure records were selected for this study. The 63 assessments selected were classified according to subjects' noise exposure into three levels: 79 to $84.9 \mathrm{~dB}(\mathrm{~A}), 85$ to $89.9 \mathrm{~dB}(\mathrm{~A})$, and 90 to $98.9 \mathrm{~dB}(\mathrm{~A})$. Occurrences of hearing loss and significant auditory threshold shifts were assessed, in each group. Results: Differences in the mean thresholds were observed in all test frequencies among the different groups. A significant correlation was found between occurrence of noise-induced hearing loss and duration of noise exposure (in years) at the present industrial facility $(\mathrm{R}=0.373 ; \mathrm{p}=0.079)$. Significant auditory threshold shifts were found in all three levels of noise exposure. Conclusion: The findings of the present study suggest the existence of an association between significant auditory threshold shifts in workers and the years of exposure to low risk noise levels.
\end{abstract}

Keywords: Permissible limits; Legislation as topic; Hearing loss, noise-induced hearing loss; Occupational exposure; Noise, occupational 


\section{REFERÊNCIAS}

1. Gonçalves CG. Saúde do trabalhador: da estruturação à avaliação de programas de preservação auditiva. São Paulo: Roca; 2009.

2. Regazzi RD, Servilieri KM, Sartorelli EM, Lima LB, Freitas EQ, Bastos DM. O risco de danos auditivos induzido por ruído ambiental, substâncias ototóxicas e o nexo causal. Mundo Saúde. 2005;29(2):243-51.

3. American Conference of Government Industrial Hygienists - ACGIH. Threshold limit values for chemical substances and physical agents in the workroom environment with intended changes for 1977. Cincinnati (OH): ACGIH; 1976.

4. Brasil. Ministério do Trabalho. Portaria n. 3214 de 08 de junho de 1978. Aprova as normas regulamentadoras do Capítulo V, Título II, das leis do trabalho, relativas a segurança e medicina do trabalho. Diário Oficial da República Federativa do Brasil, Brasília (DF); 1978 Jul 6.

5. Occupational Safety and Health Administration - OSHA. Occupational noise exposure: hearing conservation amendment, 46 Fed. Reg. 40784179. Washington (DC): OSHA;1981.

6. Suter AH. The hearing conservation amendment: 25 years later. Noise Health. 2009;11(42):2-7.

7. Agência Européia para a Segurança e Saúde do Trabalhador. Directiva 89/391/CEE do Conselho - "Directiva-quadro" relativa à adopção de medidas que se destinam a promover a melhoria da segurança e da saúde dos trabalhadores no local de trabalho. Bruxelas; 2003 [Internet] [citado 2011 Jun 17]. Disponível em: http://osha.europa.eu/pt/topics/ maintenance/maintenance-european-legislation.

8. Brasil. Ministério do Trabalho. Fundacentro. Norma de higiene ocupacional para avaliação da exposição ocupacional ao ruído (NHO 01) [Internet]. 1999 São Paulo: Fundacentro;1999 [citado 2007 Abr 7]. Disponível em: www.fundacentro.gov.br.

9. National Institute for Occupational Safety and Health. Criteria for a recommended standard: occupational noise exposure No. 98-126 [Internet]. Atlanta (GA): NIOSH; 1998 [cited 2007 Mar 8]. Available from: http://www.cdc.gov/NIOSH/docs/98-126/chap3.html.

10. Sarda ES, Ruiz, RC, Kirtsching G. Tutela jurídica dos empregados de frigoríficos: considerações dos serviços públicos. Acta Fisiátrica. 2009;16(2):59-65.

11. Brasil. Ministério do Trabalho. Secretaria de Segurança e Saúde no Trabalho Portaria n. 19, de 9 de abril de 1998. Estabelece diretrizes e parâmetros mínimos para a avaliação e acompanhamento da audição dos trabalhadores expostos a níveis de pressão sonora elevados. Diário Oficial da República Federativa do Brasil, Brasília (DF); 1998 Abr 9; Seção I:64-66.

12. Kähari K, Zachau G, Eklöf M, Sandsjö L, Möller C. Assessment of hearing and hearing disorders in rock/jazz musicians. Int J Audiol 2003;42:279-88.
13. Rabinowitz PM, Galusha D, Dixon-Ernst C, Slade MD, Cullen MR. Do ambient noise exposure levels predict hearing loss in a modern industrial cohort? Occup Environ Med. 2007;64(1):53-9.

14. Gonçalves CG, Iguti AM. Análise de programas de preservação da audição em quatro indústrias metalúrgicas de Piracicaba, São Paulo, Brasil. Cad Saúde Pública. 2006;22(3):609-18.

15. Kwitko A. Coletânea: PAIR, PAIRO, RUÍDO, EPI, EPC, PCA, CATA, perícias, reparação e outros tópicos sobre audiologia ocupacional. São Paulo: LTr; 2001.

16. Abel SM, Haythornthwaite CA. The progression of noise-induced hearing loss. A survey of workers in selected industries in Canada. J Otolaryngol Suppl. 1984;13:2-36.

17. Rabinowitz PM. Noise-induced hearing loss. Am Fam Physician. 2000;61(9):2749-56, 2759-60.

18. Lutman ME. What is the risk of noise-induced hearing loss at $80,85,90$ $\mathrm{dB}(\mathrm{A})$ and above? Occup Med (Lond). 2000;50(4):274-5.

19. Daniell WE, Swan SS, McDaniel MM, Camp JE, Cohen MA, Stebbins JG. Noise exposure and hearing loss prevention programmes after 20 years of regulations in the United States. Occup Environ Med. 2006;63(5):343-51.

20. Prince MM, Gilbert SJ, Smith RJ, Stayner LT. Evaluation of the risk of noise-induced hearing loss among unscreened male industrial workers. J Acoust Soc Am. 2003;113(2):871-80.

21. Adera T, Gullickson GM, Helfer T, Wang L, Gardner JW. Should the audiometric database analysis method (draft ANSI S12.13-1991) for evaluating the effectiveness of hearing conservation programs be accepted as a US national standard?. J Am Acad Audiol. 1995;6(4):302-10.

22. Simpson TH, Stewart M, Kaltenbach JA. Effects of audiometric threshold step size on proposed ANSI S12.13 outcomes for characterizing hearing conservation program effectiveness. J Am Acad Audiol. 1993;4(4):258-63.

23. Harger MR, Barbosa-Branco A. Efeitos auditivos decorrentes da exposição ocupacional ao ruído em trabalhadores de marmorarias no Distrito Federal. Rev Assoc Med Bras. 2004;50(4):396-9.

24. Franks JR, Davis RR, Kreig EF Jr. Analysis of a hearing conservation program data base: factors other than workplace noise. Ear Hear. 1989;10(5):273-80.

25. Petrick ME, Royster LH, Royster JD, Reist P. Comparison of daily noise exposures in one workplace based on noise criteria recommended by ACGIH and OSHA. Am Ind Hyg Assoc J. 1996;57(10):924-8.

26. Suter AH. Construction noise: exposure, effects, and the potential for remediation; a review and analysis. AIHA J (Fairfax, Va). 2002;63(6):768-89.

27. Mahendra PKV, Sridhar V. The relationship between noise frequency components and physical, physiological and psychological effects of industrial workers. Noise Health. 2008;10(40):90-8. 